\title{
FUENTES PARA UN ESTUDIO \\ DE LA LITERATURA ECUATORIANA
}

\author{
POR \\ ANTONIO SACOTO \\ New York City College \& Graduate Center
}

En este estudio señalaremos las obras críticas o relacionadas con la crítica, como el ensayo interpretativo de la cultura y la literatura, que deben ser consultadas para una comprensión y análisis de la literatura ecuatoriana de, más o menos, los últimos cincuenta años.

Para cumplir nuestro propósito, nos proponemos regirnos por el siguiente esquema:

1. General. Estudios generales e historia de la literatura.

2. Estudios sobre determinados autores:

2.1. En el exterior.

2.2. En el Ecuador.

3. Estudios por géneros.

3.1. La narrativa.

3.2. La poesía.

3.3. E1 teatro.

3.4. El ensayo.

4. Revistas literarias.

1. General. Estudios generales e historia de la literatura

La Historia de la literatura ecuatoriana, de Isaac Barrera, es la más completa en su género; se publicó en 1944. Sigue siendo obra de consulta obligatoria, principalmente en lo que va hasta la generación del $30^{1}$.

${ }^{1}$ Hay una nueva edición de Isaac Barrera, Historia de la literatura ecuatoriana (Quito: Libresa, 1979), 1.317 pp. Incluye un índice onomástico. 
Panorama de la literatura ecuatoriana (1936), de Augusto Arias. En su tiempo «llenó una necesidad impostergable: la de un texto rápido, lo más completo posible, de literatura nacional».

Pensamiento y literatura del Ecuador (1972), de Galo René Pérez, es historia crítica y antología. Obra madura, de mucho trabajo y meditación, donde el autor conjuga con éxito su saber de nuestra cultura y literatura. Cuidadoso de su estilo, va hilvanando la historia literaria de nuestro país, con muchos aciertos, pero también con algunos errores, como el de dejar fuera de este estudio a Benjamín Carrión, indicativo del poco valor que se da al ensayo ecuatoriano como género literario. Sobre este aspecto, en realidad se citan sólo tres ensayistas del siglo xx: Gonzalo Zaldumbide, Augusto Arias y Darío Moreira. Igualmente nos llama la atención que en las apreciaciones de dicha obra no haya fuentes de consulta y tampoco - como la disciplina exige- se dé una bibliografía. Sea de ello lo que fuere, y a pesar de las omisiones anotadas, la obra es valiosa y de consulta indispensable.

Hernán Rodríguez Castelo merece un aparte en la crítica ecuatoriana. El voltumen 100 de la Biblioteca de Autores Ecuatorianos publica Literatura ecuatoriana, que confirma la idoneidad y seriedad críticas del estudioso de nuestras letras. Este volumen versa sobre los siglos XvI y xvi (también carece de bibliografía). Los estudios prólogos de esta colección, escritos en su mayoría por Rodríguez Castelo, a pesar del reducido espacio, tienen juicios certeros ${ }^{2}$.

Su Literatura ectatoriana (1830-1980), publicada en 1980, es libro ligero, a veces apasionado, quizá demasiado apasionado en sus juicios sobre algunos autores contemporáneos. Sin embargo, es obra de consulta rápida y valiosa. Literatura en la audiencia de Quito Siglo XVII (1980) es, sin duda, lo más amplio y profundo que se ha escrito sobre el período colonial. Esta obra confirma una vez más la seriedad, disciplina, metodología y saber del crítico.

Entre la ira y la esperanza (1976), de Agustín Cueva, es el ejemplo más nítido del cuestionamiento cultural, literario, histórico, etc. Cueva recorre las aristas culturales buscando darles una dimensión más amplia dentro del contexto, además del histórico-literario, el sociológico y/o político-ideológico. Es verdaderamente remozador leer esas páginas, más que apasionadas, polémicas. Agustín Cueva, con gran acervo cultural, con una

${ }^{2}$ Hernán Rodríguez Castelo es también autor de las siguientes obras: Claves y secretos de la literatura infantil y juvenil (1981), Léxico sexual ecuatoriano y latinoamericano (1980) y Teatro ecuatoriano (Visiones de conjunto y selección de las mejores piezas. Clásicos Ariel, núms. 17, 36, 55 y 98). 
metodología rigurosa (el materialismo dialéctico) y una visión sin teparos ni vacilaciones, acomete su tarea de revalorar la literatura y cultura ecuatorianas. Sus juicios levantaron polvo, que aún no se ha asentado, pero la vigencia de su obra dentro y fuera del Ecuador es testimonio de su valor. Su estilo incisivo, lapidario y lleno de ironía se aviene al tratamiento del tema. En esta vertiente, él es autor, además, de La literatura ecuatoriana (1968), libro de apenas 68 páginas, pero valioso; igual Jorge Icaza, donde señala las claves para el estudio del gran novelista ecuatoriano. Lecturas $y$ rupturas (1986) es el libro más reciente de Agustín Cueva, en el que recoge artículos y ponencias sobre literatura ecuatoriana. De sumo interés son: «E1 método materialista histórico aplicado a la periodización de la historia de la literatura ecuatoriana» $\mathrm{y}$ «En pos de la historicidad perdida. Contribución al debate sobre la literatura indigenista del Ecuador» ${ }^{3}$.

El Banco Central del Ecuador ha tenido el acierto de publicar una serie de obras (antologías críticas) sobre algunos clásicos de nuestra literatura y cultura. Dichas obras vienen precedidas de extensos y bien documentados prólogos; citamos como ejemplos los siguientes:

Pensamiento romántico ecuatoriano (1980), Prólogo de Rodolfo Agoglia.

Pensamiento sociológico (1981), Prólogo de Rafael Quintero.

Psicología y sociología del pueblo ecuatoriano (1979), Prólogo de Andrés Roig.

El pensamiento filosófico social (1979), Prólogo de Hernán Malo 4.

Hay muchos otros libros publicados por el Banco Central en la colección Pendoneros. Todos estos libros conforman las exigencia profesionales.

La pluma y el cetro (1977), de Juan Valdano, es un aporte al estudio de las generaciones en el Ecuador. El libro es serio.

Michael Handelsman, estudioso norteamericano, ha incursionado en un tema postergado en la crítica ecuatoriana: la mujer como escritora y como tema. Su sugestivo y bien documentado libro Amazonas y artistas (1978), dos volúmenes, con el subtítulo «un estudio de la prosa de la mujer ecuatoriana», tiene un enfoque moderno y un empaque cosmopolita. El éxito de este libro estimuló la publicación de su antología Diez escritoras ecuatorianas y sus cuentos (1982).

${ }^{3}$ Agustín Cueva ha publicado además: El proceso de dominación politica en el Ecuador (1972), El desarrollo del capitalismo en América Latina (1977) y Teoría social y procesos politicos en América Latina (1979).

${ }^{4}$ Mencionamos solamente algunos títulos de esta serie, que está ya en el número 28. 
2. ESTUdios SOBRE DETERMINADOS AUTORES

(sólo mencionamos los títulos de la obra y el autor)

2.1. En el exterior ${ }^{5}$

Enrique Anderson Imbert, El arte de la prosa en Juan Montalvo (1948).

Philip Louis Astuto, Eugenio Espejo, reformador de la Ilustración (1969).

Kent B. Mecum, El idealismo práctico de Vicente Rocafuerte (1975).

Theodore Sackett, El arte de la novelística de Jorge Icaza (1974).

Enrique Ojeda, Jorge Carrera Andrade (1971).

Antonio Sacoto, Juan Montalvo, el escritor y el estilista (1973).

Gerardo Luzuriaga, Del realismo al expresionismo: el teatro de Aguilera Malta (1971).

Clementine Rabassa, En torno a Aguilera Malta (1981).

María Valverde, La narrativa de Aguilera Malta (1981).

Dario Lara, Montalvo en París (1983), dos tomos.

Humberto Robles, Testimonio y tendencia mítica en la obra de José de la Cuadra (1976).

\subsection{En el Ecuador}

María Rosa Crespo, Tras las huellas recogidas de César Dávila Andrade (1980).

Juan Valdano, Léxico y símbolo en Juan Montalvo (1981).

Jorge Isaac Cazorla, Metanoia de Juan Montalvo, s. f. (1980?).

Manuel Corrales Pascual, Jorge Icaza: frontera del relato indigenista (1974).

\section{ESTUDIOS POR GÉNEROS}

\subsection{La narrativa}

La novela ecuatoriana (1948), de Angel Felicísimo Rojas, es obra indispensable para el estudio de este género en el Ecuador. Su valor estriba, además de los aciertos literarios, en el marco sociológico y cultural que configura el devenir de la novela.

\footnotetext{
${ }^{5}$ En el acápite «En el exterior» retınimos las obras de estudiosos de la literatura ecuatoriana que residen fuera del país.
} 
El nuevo relato ecuatoriano (1950), crítica y antología de Benjamín Carrión, es un estudio subjetivo que nace del tratamiento del tema con un afán renovador y al mismo tiempo orientador. Benjamín Carrión tiene un conocimiento amplio y de primera mano de los temas y escritores de la décado del 30; él mismo prologó algunas obras de ellos. El calor humano, la pasión, compensan la metodología.

La moderna novela ecuatoriana (1958), de Edmundo Ribadeneira, es obra también excelente en su género, principalmente por el adosamiento «de la estructura englobante (socio-político-económica) al objeto estudiado (la novela)», como anotaría Goldman y otros en Sociología de la novela.

Antonio Sacoto nos da, en 1967, The Indian in the Ecuatorian Novel, un estudio del indianismo de Juan León Mera y del indigenismo de Jorge Icaza; además se estudian otros autores de la generación del 30. Del mismo autor es La nueva novela ecuatoriana (1981), un estudio de las novelas más logradas de la década del 70, y Cinco novelas claves de la literatura hispanoamericana (1978).

La Pontificia Universidad Católica del Ecuador publica, en 1977, dos volúmenes de ensayos críticos sobre narrativa en Situación del relato ecuatoriano, y en 1979, en el centenario de la publicación de Cumandá, se publica un volumen de estudios literarios sobre dicha novela.

Libro de posta, «La narrativa actual en el Ecuador» (1983), con una selección e introducción de Miguel Donoso Pareja, es una colección de trece cuentistas contemporáneos de Ecuador.

\subsection{La poesía}

La crítica ha sabido responder al auge lírico ecuatoriano con estudios merecedores; entre los primeros se encuentran los dos volúmenes de Hernán Rodríguez Castelo Lírica ecuatoriana contemporánea (1979), precedidos de un «no-prólogo» de 30 páginas y luego una —más bien - antología de la poesía ecuatoriana a partir de César Dávila Andrade (nacido en 1919) hasta los nacidos en 1951, comprendiendo un total de 107 poetas. Desde una perspectiva internacional — quizá nacional también- para quien quiera conocer sobre poesía ecuatoriana contemporánea, 107 nombres es una cifra exagerada. Es que además no tiene sentido dedicar el mismo espacio a los buenos poetas como a los que francamente no lo son. No quisiera insistir, pero obra de tanto empeño carece de bibliografía, to que le resta valor académico. Sin embargo, y a pesar de lo anotado, la obra es de consulta imperativa para el estudio de la poesía ecuatoriana contemporánea; además, muchos de los juicios críticos de Rodríguez Castelo son certeros y ecuánimes. 
Rodrigo Pesántez Rodas ha trabajado mucho en este campo y con mucho acierto: en La nueva literatura ecuatoriana (1966) presenta a 31 poetas; es un estudio breve de cada uno de los poetas, seguido de dos o tres poemas, por lo que es, en realidad, una antología crítica (carece de bibliografía). En Siete poetas del Ecuador (s. f., ca. 1971) sigue la misma estructura: estudio introductorio del poeta y luego selecciones de poesía. Se trata nuevamente de una antología crítica (sin bibliografía). En Poesía de un tiempo (1974) estudia la generación del 60.

Antología de la poesía cuencana (1981), de Antonio Lloret Bastidas, sigue igual tratamiento.

Gonzalo Ramón nos da La poesía ecuatoriana, con el subtítulo de «Ensayo sobre Dante, Miguel Angel Zambrano», en 1969. Su acercamiento es subjetivo, pero lleno de revelaciones en lo que respecta al poeta ecuatoriano; tiene, además, estudios breves sobre otros poetas ecuatorianos.

Laura Hidalgo nos da Décimas esmeraldenas (1983), un estudio de la poesía popular transmitida oralmente en la provincia de Esmeraldas. Poco y fragmentariamente se ha escrito sobre el tema. Su libro, además de llenar este vacío, es un ejemplo de seriedad y disciplina críticas.

\subsection{El teatro}

Entre las obras que deben destacarse se encuentra Historia critica del teatro ecuatoriano (1968), de Ricardo Descalzi; es la obra más completa y de las primeras. Son seis volúmenes. En realidad se trata de una antología bien encaminada y bien documentada. Hay algunos estudios y algunos libros sobre autores en particular, como el ya mencionado de Gerardo Luzuriaga sobre Aguilera Malta, quien además acaba de publicar Bibliografía del teatro ecuatoriano (1984) del período que va de 1900 a 1982.

\subsection{El ensayo}

Benjamín Carrión es la figura más prominente en este campo en el Ecuador. Su visión sobre su país, sobre América en general, sigue las líneas de los grandes intérpretes de nuestra cultura, de nuestra historia, de nuestra realidad y de nuestras inquietudes. Con él aprendimos a amar la historia de nuestro pueblo; con él nos sentimos hermanados a Atahualpa (1934), con él estudiamos los santos del espíritu: San Miguel Unamuno (1954), Santa Gabriela Mistral (1956), García Moreno, el santo del patí- 
bulo (1959), pero principalmente José Carlos Mariátegui ${ }^{\text {; }}$; con él sentimos el dolor de la raza india postergada y explotada; con él despertamos nuestra simpatía por el apostólico y profético Las Casas. Y todo esto es lo que hace que su pensamiento sea más americanista, más universal, y ande a tono con el resto de Hispanoamérica. Es más, su pensamiento tiene vigencia hoy como ayer. Así lo habrán visto los mejicanos, que le otorgaron la presea «Benito Juárez». En su obra crítica, en sus múltiples prólogos, en sus ensayos, sienta las bases para el estudio de la cultura y la literatura ecuatorianas. Estudios posteriores, sin lugar a duda, han superado su metodología, pero en lo medular sus juicios siguen vigentes, sea para aceptarlos, meditarlos o refutarlos, como nos indican los recientes estudios de Hernán Rodríguez Castelo, Benjamín Carrión, el hombre y el escritor (1979), y de Jorge Enrique Adoum, El libro de los prólogos. Erika Silva, acertadamente, nos da algunas pautas de la importancia de Benjamín Carrión en el desarrollo de la cultura ecuatoriana en su «En torno al surgimiento de la cultura nacional» (México: FLASCO, 1980), capítulo III, parte III.

Sin embargo, algunas de las proposiciones de Carrión se cuestionan con un afán de disminuir la obra y el aporte. Por ejemplo, Fernando Tinajero cuestiona el optimismo de Carrión cuando proclama que el Ecuador nunca podría ser una potencia económica ni bélica, dada su extensión, pero sí podría ser un baluarte cultural. Tinajero, en Teoría de la cultura nacional (1986) y en Aproximaciones y distancias (1986), y Agustín Cueva, en Lecturas y rupturas (1986), rechazan tal teoría.

En Ecuador: drama y paradoja (1950), Leopoldo Benites Vinueza logra aunar paisaje y hombre en acabada simbiosis significativa de cómo la naturaleza ecuatoriana cincela sus tipos y caracteres; hay una amalgama de historia, clima y razas en la vertiente sociocultural ecuatoriana. Es un libro ensayístico lleno de datos, fuentes, referencias, documentos que pincelan el contorno preciso de nuestro Ectiador: drama y paradoja. Un zapador de la colonia (1941) es un estudio sugestivo sobre la gran figura precursora de la independencia ecuatoriana: Eugenio Espejo. En 1984 publica Francisco Eugenio Espejo, el habitante de la noche, que en realidad es el título del primer ensayo crítico; los otros dos se relacionan con José Mejía de Lequerica y Juan Montalvo. Tres figuras que le fascinaron a Benites y de quienes hace una verdadera apología.

- Benjamín Carrión es autor también de las siguientes obras: Los creadores de la nueva América (1928), Mapa de América (1930), Indice de la poesía ecuatoriana (1935), Cartas al Ecuador (1943), Nuevas cartas al Ecuador (1960), Plan del Ecuador (1977), El cuento de la patria (1967) y América dada al diablo (1981, póstuma). 
Gran parte de la obra de Plutarco Naranjo se encuentra desperdigada en revistas y periódicos. Sus estudios bibliográficos Juan Montalvo, tomos I y II (1966), son exhaustivos, serios y, sin lugar a duda, un gran aporte a los estudios montalvinos. Plutarco Naranjo es un gran conocedor de la obra total de Montalvo, un verdadero investigador y de una sensibilidad clara para desdoblar la peripecia de la obra del Cervantes de América. En Ensayos sobre Montalvo (1986) trata algunos temas de interés de la obra del ambateño, hilando lo histórico y político con lo literario.

Galo René Pérez acaba de publicar una colección de ensayos, Confesión insobornable (1986), sobre una gran variedad de temas, muchos de ellos relacionados con la cultura y la literatura ecuatorianas, con verdadero dominio de la prosa ensayística; es quizá uno de los mejores estilistas en la prosa ecuatoriana contemporánea.

Fernando Tinajero ha publicado Más allá de los dogmas (1967) y Aproximaciones y distancias (1986). Los temas del primer libro se relacionan con una definición de cultura nacional, del papel (o compromiso) del escritor, del parricidio, de la autenticidad. El segundo, del discurso ideológico, de historia y literatura, de la trayectoria del movimiento cultural (¿e ideológico?), de los problemas de su tiempo y de su generación. Los dos libros son indispensables para una comprensión del desarrollo del pensamiento ecuatoriano contemporáneo. Tinajero es, además, quien escribe el «Estudio introductorio» y hace la selección de la antología Teoría de la cultura nacional (1986).

Edmundo Ribadeneira, actual presidente de la Casa de la Cultura Ecuatoriana, da a la estampa tres libros: La condición humana a través de Frankestein y Drácula (1982), Universidad, arte y sociedad (1982) y Recopilario, el hombre y su tiempo (1984). Estos libros son recopilaciones de memorias «apasionadas para quienes escribir es una función vital..., un sudor de sangre», "plagado de violencias y contradicciones»; un acopio de sus discursos y presentaciones de autores y obras; meditaciones sobre problemas nacionales e internacionales, una recolección también de lo que alguna vez fueron artículos periodísticos, temas relacionados con educación y universidad; en fin, en esta múltiple gama temática se encuentran breves ensayos sobre cine, política, música, literatura, etc. Todas estas páginas están impregnadas por ese calor humano que da Ribadeneira a todo lo que escribe. Su estilo es breve, ágil, periodístico.

E1 estudio del ensayo ecuatoriano está casi inexplorado; lo único que hemos encontrado en el período contemporáneo es «El ensayo en la literatura ecuatoriana actual» (Cuadernos Americanos de México, julio-agosto de 1957), de Alfredo Pareja Diezcanseco, y «El ensayo y la crítica litera- 
ria ecuatoriana en la segunda mitad del siglo Xx» (Cultura, de Quito, enero-abril de 1979), de Alfonso Carrasco, y, por lo que parece, nada más.

\section{Revistas literarias}

Las revistas literarias en el Ecuador tienen vida efímera; sin embargo, la prestigiosa Letras del Ecuador, publicación de Casa de la Cultura de Quito, sigue en circulación. El guacamayo y la serpiente, revista literaria de Casa de la Cultura, núcleo del Azuay, acaba de entregar puntualmente el número 26, lo que significa un lapso de dieciocho años ininterrumpidos, pues el primer número apareció en 1969. Igual sucede con Cultura, del Banco Central de Quito, que está ininterrumpida hasta la última entrega puntual del número 22. Estas revistas llevan artículos principalmente sobre literatura ecuatoriana y gozan merecidamente de gran prestigio en el ámbito académico ecuatoriano. Desgraciadamente, debemos anotar, la difusión de estas revistas en el exterior es ínfima. Los estudios que encontramos en estas revistas son notorios por su seriedad y disciplina académica y han colaborado estudiosos de la literatura ecuatoriana de los Estados Unidos y de Europa, además de los nacionales. Cultura, por ejemplo, ha publicado las ponencias de los congresos sobre literatura ecuatoriana. Por todo ello, estas revistas son indispensables para el estudio de la literatura ecuatoriana.

Artículos sumamente interesantes, aunque de menos rigor académico, se encuentran en las revistas América, de Quito, y Letras del Guayas y Revista de la Universidad, de Guayaquil. Hay también revistas que abordan, además de lo literario, lo político-social y lo ideológico, como La revista nueva, fundada en 1972. 
